\title{
Diseño de un programa de formación básico para conseguir espirometrías de calidad
}

\author{
Joan Escarrabill, Núria Roger, Felip Burgos, Jordi Giner, Ainhoa Molins, Ricard Tresserras, \\ en nombre del Grupo de Función Pulmonar y del equipo directivo del PDMAR
}

Introducción. La espirometría forzada es una manera sencilla y no invasiva de valorar la función pulmonar. La obtención de una espirometría de calidad requiere formación, condiciones técnicas adecuadas (calibración, mantenimiento y ubicación del aparato) y colaboración del paciente. Diversos trabajos han constatado déficits formativos de los profesionales, relacionados en parte con la elevada rotación en un mismo puesto, la falta de sistemática en la incorporación de nuevos profesionales que realizan espirometrías y la ausencia de planes de evaluación periódica de competencias.

Materiales y métodos. En el marco del Plan Director de Enfermedades del Aparato Respiratorio (PDMAR) se ha diseñado un programa formativo mínimo teórico-práctico de 16 horas de duración, basado en el programa de formación del National Institute for Occupational Safety and Health y de las iniciativas que surgen en el marco de la European Respiratory Society. Durante el año 2010 se realizaron 13 cursos en los que participaron 307 profesionales.

Resultados. Las diferencias observadas entre la evaluación de conocimientos previos y la evaluación final fue estadísticamente significativa $(p<0,001)$.

Conclusiones. La formación debe organizarse de modo descentralizado y con base territorial con el fin de garantizar el acceso de todos los profesionales que deben realizar espirometrías. La espirometría de calidad únicamente podrá conseguirse si se integran todos los elementos clave (formación, aspectos técnicos e interpretación), por lo que es imprescindible la interoperabilidad entre los diferentes ámbitos asistenciales.

Palabras clave. Espirometría. Espirometría de calidad. Formación.

\section{Design of a basic training program to get quality spirometry}

Introduction. The spirometry is a simple and noninvasive test to assess lung function. Obtaining a spirometry of quality requires training, appropriate technical conditions (calibration, maintenance and location of the device), and patient cooperation. Several studies have found professional training deficits related to a high turnover in the same place, the lack of systematic training when new professionals performing spirometry are incorporated and the lack of competences' periodic evaluation.

Materials and methods. The Master Plan for respiratory diseases (PDMAR) has designed a minimum practical/theoretical training program (16 hours) based on the training program of the National Institute for Occupational Safety and Health and the European Respiratory Society initiatives. In 2010, 13 courses have been offered to 307 professionals.

Results. The differences observed between the initial assessment and post evaluation knowledge was statistically significant $(p<0.001)$.

Conclusions. Training must be organized in a decentralized and territorial basis in order to ensure access for all professionals performing spirometry. Moreover, a spirometry of quality will only be achieved when all key components are integrated (training, technical aspects and interpretation) which requires interoperability between different levels of care.

Key words. Lung function test. Spirometry of quality. Training.

\section{Introducción}

El impacto de las enfermedades respiratorias en nuestro medio resulta muy elevado tanto desde el punto de vista de la mortalidad (tercera causa de muerte) [1] como en lo que se refiere al consumo de recursos (en nuestro medio causan el 10\% de los ingresos hospitalarios y comportan el gasto de alrededor del 8,5\% del presupuesto total del Servei Català de la Salut) [2]. La enfermedad pulmonar obstructiva crónica (EPOC) afecta al 10\% de personas de más de 40 años $[3,4]$ y constituye la afectación respiratoria no neoplásica con mayor carga asistencial.
Pla Director de les Malalties de I'Aparell Respiratori, PDMAR; Institut d'Estudis de la Salut; Departament de Salut (J. Escarrabill). Servicio de Neumología; Consorci Hospitalari de Vic (N. Roger). Servicio de Neumología, ICT; Hospital Clínic; IDIBAPS (F. Burgos). Servicio de Neumología; Hospital de la Santa Creu i Sant Pau (J. Giner). Institut $d^{\prime}$ Estudis de la Salut (A. Molins). Direcció General de Regulació, Planificació i Recursos Sanitaris; Departament de Salut; Barcelona, España (R. Tresserras).

Grupo de Función Pulmonar: B. Avilés (Hospital de Palamós); F. Burgos (Hospital Clínic, Barcelona); J. Clotet (EAP Ponts, Lleida); J. Delclós (University of Texas School of Public Health, Estados Unidos); J. Escarrabill (PDMAR, Institut d'Estudis de la Salut, Barcelona); J. Giner (Hospital de la Santa Creu i Sant Pau, Barcelona); M.A. Llauger (EAP Encants, CAP Maragall, Barcelona); R. Hervàs (CAE Dr. Barraquer, Sant Adrià del Besòs, Barcelona); F. Martín (EAP Tarragona, CAP Jaume I, Tarragona); C. Martín, A. Moreno (Hospital Vall d'Hebron, Barcelona); F. Moya (TIC Salut); N. Roger (Consorci Hospitalari de Vic); C. Rubies, T. Salas (Departament de Salut, Área TICS).

Equipo directivo del PDMAR: J. Escarrabill (Institut d'Estudis de la Salut, Barcelona); C. Hernández (Hospital Clínic, Barcelona); J. Jiménez (CatSalut, Barcelona) M.A. Llauger (EAP Encants, CAP Maragall, Barcelona); N. Roger (Consorci Hospitalari de Vic); A. Rosas (Direcció General de Regulació, Planificació i Avaluació; Departament de Salut, Barcelona); E. Saltó (Direcció General de Salut Pública, Barcelona); R. Tresserras (Direcció General de Regulació, Planificació i Recursos Sanitaris; Departament de Salut, Barcelona).

Correspondencia:

Dr. Joan Escarrabill. Pla Director de les Malalties de l'Aparell Respiratori (PDMAR). Institut d'Estudis de la Salut. Roc Boronat, 81-95, 1.‥ E-08005 Barcelona. 


\section{E-mail: jescarrabill@gencat.cat. Conflicto de intereses: No declarado. \\ Conflict of interests None declared.}

(c) 2012 Educación Médica
Las enfermedades respiratorias pueden diagnosticarse mediante una prueba no invasiva y de bajo coste: la espirometría forzada. Existe un amplio consenso sobre la estandarización de la prueba $[5,6]$, pero como su realización requiere la colaboración del paciente, los resultados obtenidos son muy variables. Para conseguir una prueba de calidad se requiere disponer de aparatos fiables y calibrados, un ambiente apropiado, profesionales entrenados y pacientes adecuadamente informados y dispuestos a colaborar. Naberan et al [7] muestran que la falta de entrenamiento de los profesionales es un factor muy importante en el número y calidad de las espirometrías realizadas en atención primaria. Hueto et al [8] observan que en Navarra, a pesar de que la mayor parte de los centros de atención primaria tienen espirómetro, el 22\% no lo utilizan. Este hecho no quiere significar que la espirometría en la atención primaria no sea posible $[9,10]$. Uno de los problemas más frecuentes, además de los déficits formativos, es el alto grado de rotación en los puestos de trabajo de los profesionales de enfermería entrenados.

El infradiagnóstico constituye uno de los problemas más graves en el manejo de las enfermedades respiratorias, especialmente de la EPOC [11], que junto al 'mal diagnóstico' $[12,13]$ se observa incluso en el medio hospitalario [14]. La reducción del infradiagnóstico y el diagnóstico apropiado requieren intervenciones específicas, pero sobre todo garantizar el acceso a una espirometría de calidad [15-17].

El Departament de Salut de la Generalitat de Catalunya se sirve de los planes directores [18] para acercar los planteamientos estratégicos a los operativos con el fin de mejorar la atención al paciente. En este contexto, uno de los objetivos prioritarios del Plan Director de Enfermedades del Aparato Respiratorio (PDMAR) es garantizar al clínico el acceso a una espirometría de calidad independientemente del ámbito asistencial en el que trabaje [19]. Dado que se ha constatado el déficit formativo de muchos profesionales, parece razonable plantearse el diseño de un programa formativo general, entendiéndolo como una estrategia para conseguir la espirometría de calidad.

\section{Materiales y métodos}

La propuesta formativa se ha diseñado a partir del programa de formación del National Institute for Occupational Safety and Health (NIOSH) [20] y de las iniciativas que surgen en el marco de la European Respiratory Society [21]. La formación básica debe comprender aspectos teóricos y prácticos en un for- mato de cursos de 2-4 días, en función de la distribución de las clases. A través de un grupo de trabajo multidisciplinar se ha consensuado un programa teórico-práctico de 16 horas que se recomienda desarrollar a lo largo de dos días consecutivos. Los contenidos del programa se resumen en la tabla.

Los organizadores de los cursos recibieron, junto al programa, el desarrollo de los contenidos en versión PowerPoint, para que pudieran adaptarlos a sus necesidades.

\section{Resultados}

Durante el año 2010 se acreditaron 13 cursos de formación que siguieron el programa formativo diseñado en el marco del PDMAR. Once cursos fueron 'generales' (según el programa descrito en la tabla), uno se centró en pediatría y otro fue un curso de 'formación de formadores'. En total participaron 307 profesionales: enfermeras (68\%), médicos (2\%), farmacéuticos (21\%), enfermeras pediátricas $(8 \%)$ y otros $(1 \%)$.

\section{Valoración de conocimientos}

Los asistentes a los cursos realizaron una evaluación inicial de conocimientos mediante 10 preguntas sobre aspectos básicos de la realización de la espirometría, que debían responder antes de iniciar el curso.

La media de puntos obtenida fue de $5 \pm 2$; el $37 \%$ de los alumnos alcanzó una puntuación inferior a 5 puntos.

Al final del curso se realizó una nueva evaluación con el mismo cuestionario, al cual se añadieron cinco preguntas más. El resultado obtenido (sobre 10 puntos) fue de $8 \pm 2$ puntos y únicamente el 15\% de los alumnos obtuvo una puntuación inferior a 5 puntos. Las diferencias observadas entre la puntuación inicial y la final fueron estadísticamente significativas $(p<0,001)$.

\section{Valoración de los alumnos}

La valoración cualitativa de los cursos se resume en la figura.

Las puntuaciones más bajas en los aspectos organizativos y de calidad del material se relacionan con las valoraciones de los primeros cursos, en los que todavía se utilizaban materiales provisionales. A partir del cuarto curso, en el que ya se dispuso del material definitivo, las valoraciones medias fueron superiores a 8,7 . 
Tabla. Contenidos del programa.

\begin{tabular}{|c|c|c|c|}
\hline & & Contenido & Duración \\
\hline Módulo 1 & $\begin{array}{l}\text { Anatomía y fisiología pulmonar } \\
\text { relacionada con la espirometría }\end{array}$ & $\begin{array}{l}\text { Sistema respiratorio: aspectos básicos de anatomía y fisiología } \\
\text { Mecanismos de la respiración: la bomba respiratoria, aspectos básicos de la mecánica respiratoria }\end{array}$ & $1,5 \mathrm{~h}$ \\
\hline Módulo 2 & $\begin{array}{l}\text { Historia de la espirometría. } \\
\text { La espirometría en neumología }\end{array}$ & $\begin{array}{l}\text { Breve reseña histórica de la espirometría. } \\
\text { La espirometría en las normativas de las enfermedades respiratorias (asma y EPOC) }\end{array}$ & $0,5 \mathrm{~h}$ \\
\hline Módulo 3 & Aspectos básicos de la espirometría & $\begin{array}{l}\text { Definición de espirometría } \\
\text { Tipos de espirómetro } \\
\text { Cómo hacen las medidas } \\
\text { Representación de la espirometría } \\
\text { Volumen/tiempo } \\
\text { Flujo/volumen } \\
\text { Alteraciones funcionales pulmonares (obstrucción y no obstrucción) en la espirometría } \\
\text { Control y calibración de los espirómetros }\end{array}$ & $1,0 \mathrm{~h}$ \\
\hline
\end{tabular}

Indicaciones y limitaciones de la espirometría

Valores de referencia

Formas de medición de los parámetros observados respecto a la referencia

Módulo 4 Técnica de la espirometría I Preparación del sujeto para realizar la espirometría Instrucciones

Realización de la prueba

Criterios de aceptabilidad y reproducibilidad de las maniobras

Número de maniobras a realizar

¿Qué hay que mirar de cada maniobra?

Gráfica

Números

Módulo 5 Técnica de la espirometría II

Inicio de la maniobra, extrapolación retrógrada

Transcurso de la maniobra

Final de la maniobra

Maniobra inspiratoria

Utilización de los rangos de referencia (LMN, LLN)

Componentes de un programa de control de calidad

Módulo 6 Control de calidad

Verificaciones de la calibración y otras medidas de control de calidad de los equipos

Contaminación de los equipos y control de las infecciones

Práctica conjunta con todos los asistentes. Realización de espirometrías en formato de rol-play con diferentes circunstancias

Módulo 7 Práctica en la espirometría

Práctica en grupos pequeños. Práctica donde se hacen espirometrías a todos los participantes

Clasificación de la espirometría

Errores más frecuentes: del sujeto y del técnico

Valoración de les maniobras

Módulo 9 Espirometría en niños

Valoración de les maniobres inspirométricas

Diferencia entre los resultados de la espirometría y de las medidas del flujo máximo (PFR)

Prueba broncodilatadora

Módulo 10 Otras aplicaciones de la espirometría

$$
\text { Realización }
$$

Valoración

Pruebas de provocación bronquial

Otras aplicaciones

Módulo 11 Interpretación de la espirometría

Sesión práctica en la que se valoran conjuntamente los aspectos gráficos y numéricos de las maniobras espirométricas 
Figura. Valoración cualitativa de los cursos.

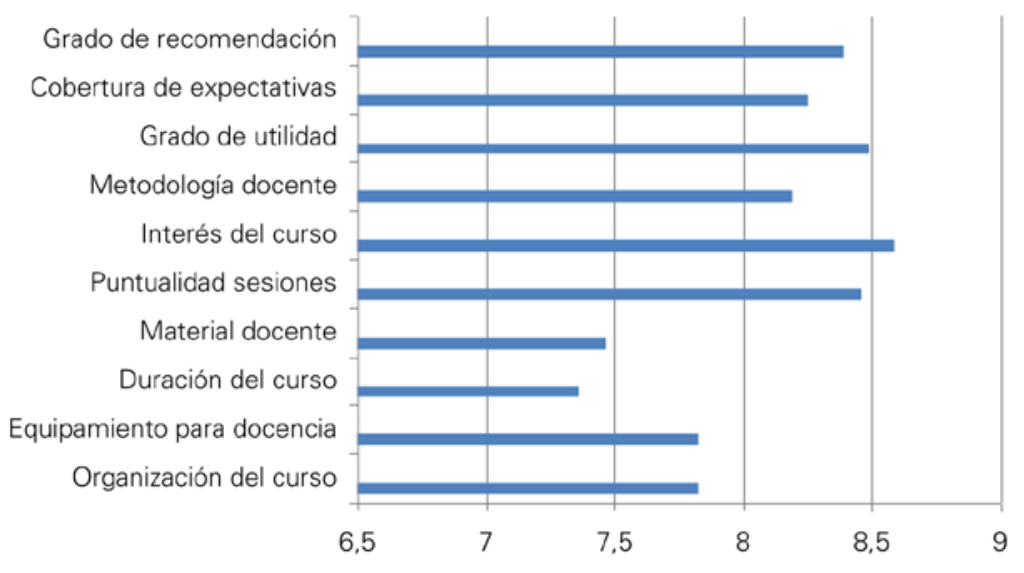

\section{Discusión}

La propuesta formativa es factible. En ningún caso el curso propuesto se debió anular por problemas técnicos, aunque en algunos casos desde el PDMAR se colaboró facilitando el contacto con el profesorado experto.

Este tipo de formación plantea tres problemas importantes:

- Si los cursos se ofrecen de una manera espontánea es poco probable que se produzca una cobertura homogénea del territorio.

- Se requiere la implicación de los proveedores de servicios sanitarios para garantizar que las personas preparadas serán las que se destinarán a los lugares de trabajo en los que se realizan las espirometrías (lo cual no quiere decir que hagan espirometrías de una manera exclusiva).

- La formación básica no garantiza el mantenimiento de las habilidades, especialmente en los casos en los que los profesionales realizan pocas pruebas (o las realizan de una manera intermitente) [22].

En este sentido, desde el PDMAR se trabaja para que el Servei Català de la Salut incluya en el contrato de servicios la formación en espirometría, promoviendo la realización de cursos en los territorios donde todavía no se ha realizado esta formación.

La formación de profesionales que han de realizar espirometrías debería considerar estas características:
- Reglada.

- Descentralizada.

- Con un gran componente práctico.

- Con cobertura territorial.

- Debe contemplar la recertificación (o revalidación de competencias) [23].

- Formación continuada para mantener la calidad y programas de autocontrol de lo que cada uno sabe (programa que de una manera anónima permita contrastar los conocimientos personales).

Estos objetivos no se conseguirán con programas de formación puntuales y centralizados. Es necesario promover la formación de formadores en cada territorio para que puedan asumir esta perspectiva más amplia de la formación, que va más allá de la realización de cursos.

Steenbruggen et al [24] señalan que la formación es un elemento crucial para mejorar la práctica de la espirometría, pero en ningún caso es el objetivo final. Borg et al [22] presentan datos acerca de que la formación de 14 horas no es suficiente para garantizar espirometrías de calidad a los cinco meses de la formación y ponen énfasis en la necesidad de efectuar una revisión activa de las espirometrías para el mantenimiento de las competencias. No tendría demasiado sentido separar la formación de la calidad. Formamos profesionales para que sean capaces de obtener espirometrías de calidad. Ese es el objetivo: el acceso fácil por parte del clínico a espirometrías de calidad. Por lo tanto, los proveedores de servicios deberían promover sistemas de financiación que garantizaran la cobertura de las necesidades (es decir, incrementar el número de espirometrías) con garantías de calidad (pagar por pruebas bien hechas).

Los elementos para conseguir una espirometría de calidad son:

- Disponer de aparatos fiables y calibrados.

- Programas formativos adecuados para los profesionales que deben realizar espirometrías y que incluyan: formación básica, formación avanzada, formación específica para la edad pediátrica (incluida en el módulo general y, además, mediante un módulo específico pediátrico), formación de formadores y programas de revalidación de competencias.

- Los programas formativos deben incluirse en la compra de servicios a los proveedores.

- La interoperabilidad entre los distintos ámbitos asistenciales es imprescindible para permitir el control de calidad.

- Formación para la correcta interpretación de resultados [25]. 
- Formulación de objetivos individuales que incentiven a los profesionales a la realización de espirometrías de calidad: porcentaje de pacientes con EPOC y otras enfermedades respiratorias como el asma, en los que se ha practicado espirometría en un periodo dado.

- Definición de los criterios mínimos que deben reunir los centros sanitarios para realizar correctamente las espirometrías: identificación de los responsables de la calibración y el mantenimiento, condiciones mínimas del lugar donde se realizan las espirometrías y capacitación de los profesionales que deben realizar espirometrías.

Un tema abierto al debate se centra en la organización del control de calidad. En los estudios multicéntricos está claro que la mejor opción es un control de calidad centralizado [26], pero se requieren más estudios para poder aplicar este criterio a la práctica cotidiana.

En un futuro inmediato, para conseguir estos objetivos deberán utilizarse tecnologías de la información y de la comunicación que permitan la formación online y proporcionen soporte a la toma de decisiones, tanto organizativas y técnicas (calibración o control de calidad, por ejemplo) como asistenciales (indicación e interpretación).

\section{Bibliografía}

1. Lopez AD, Mathers CD, Ezzati M, Jamison DT, Murray CJ. Global and regional burden of disease and risk factors, 2001: systematic analysis of population health data. Lancet 2006; 367: 1747-57.

2. Gibert R, Brosa M. Avaluació de la participació del plans directors en la despesa sanitària del CatSalut. Estudis d'Economia de la Salut. Barcelona: Generalitat de Catalunya, Departament de Salut; 2006.

3. Halbert RJ, Natoli JL, Gano A, Badamgarav E, Buist AS, Mannino DM. Global burden of COPD: systematic review and meta-analysis. Eur Respir J 2006; 28: 523-32.

4. Miravitlles M, Soriano JB, García-Río F, Muñoz L, DuránTaulería E, Sánchez G, et al. Prevalence of COPD in Spain: impact of undiagnosed COPD on quality of life and daily life activities. Thorax 2009; 64: 863-8.

5. Ferguson GT, Enright PL, Buist AS, Higgins MW. Office spirometry for lung health assessment in adults: a consensus statement from the National Lung Health Education Program. Chest 2000; 117: 1146-61.

6. Levy ML, Quanjer PH, Booker R, Cooper BG, Holmes S, Small I; General Practice Airways Group. Diagnostic spirometry in primary care: proposed standards for general practice compliant with American Thoracic Society and European Respiratory Society recommendations -a General Practice Airways Group (GPIAG) document, in association with the Association for Respiratory Technology \& Physiology (ARTP) and Education for Health. Prim Care Respir J 2009; 18: 130-47.

7. Naberan K, De la Roza C, Lamban M, Gobartt E, Martín A, Miravitlles M. Utilización de la espirometría en el diagnóstico y tratamiento de la EPOC en atención primaria. Arch Bronconeumol 2006; 42: 638-44.
8. Hueto J, Cebollero P, Pascal I, Cascante JA, Eguía VM, Teruel F, et al. Spirometry in primary care in Navarre, Spain. Arch Bronconeumol 2006; 42: 326-31.

9. Poels PJ, Schermer TR. Good wine needs no bush: quality spirometry in a primary care setting is possible. Arch Bronconeumol 2007; 43: 473.

10. Yawn BP, Enright PL, Lemanske RF Jr, Israel E, Pace W, Wollan P, et al. Spirometry can be done in family physicians' offices and alters clinical decision in management of asthma and COPD. Chest 2007; 132: 1162-8.

11. Soriano JB, Ancochea J, Miravitlles M, García-Río F, DuránTaulería E, Muñoz L, et al. Recent trends in COPD prevalence in Spain: a repeated cross-sectional survey 1997-2007. Eur Respir J 2010; 36: 758-65.

12. Menezes AM, Pérez-Padilla R, Jardim JR, Muiño A, López MV, Valdivia G, et al; PLATINO Team. Chronic obstructive pulmonary disease in five Latin American cities (the PLATINO study): a prevalence study. Lancet 2005; 366: 1875-8.

13. Proyecto Latinoamericano de Investigación en Obstrucción Pulmonar (PLATINO). URL: http://www.platino-alat.org. [02.02.2011].

14. Pellicer-Císcar C, Soler-Cataluña JJ, Andreu-Rodríguez AL, Bueso-Fabra J; en representación del Grupo EPOC de la Sociedad Valenciana de Neumología. Calidad del diagnóstico de enfermedad pulmonar obstructiva crónica en el ámbito hospitalario. Arch Bronconeumol 2010; 46: 64-9.

15. Clotet J, Gómez-Arbonés X, Ciria C, Albalad JM. Spirometry is a good method for detecting and monitoring chronic obstructive pulmonary disease in high-risk smokers in primary health care. Arch Bronconeumol 2004; 40: 155-9.

16. Soriano JB, Zielinski J, Price D. Screening for and early detection of chronic obstructive pulmonary disease. Lancet 2009; 374: 721-32.

17. Schirnhofer L, Lamprecht B, Firlei N, Kaiser B, Buist AS, Halbert RJ, et al. Using targeted spirometry to reduce non-diagnosed chronic obstructive pulmonary disease. Respiration 2011; 81: 476-82.

18. Tresserras R. Planificación según prioridades de salud. Criterios de planificación relacionados con los planes directores. Med Clin (Barc) 2008; 131 (Supl 4): 42-6.

19. Levy ML, Quanjer PH, Booker R, Cooper BG, Holmes S, Small I. Diagnostic spirometry in primary care: proposed standards for general practice compliant with American Thoracic Society and European Respiratory Society recommendations -a General Practice Airways Group (GPIAG). Prim Care Respir J 2009; 18: 130-47.

20. Centers for Disease Control and Prevention. Spirometry Training Program. URL: http://www.cdc.gov/niosh/topics/ spirometry/training.html. [01.03.2011].

21. Miller MR, Crapo R, Hankinson J, Brusasco V, Burgos F, Casaburi R, et al; ATS/ERS Task Force. General considerations for lung function testing. Eur Respir J 2005; 26: 153-61.

22. Borg BM, Hartley MF, Fisher MT, Thompson BR. Spirometry training does not guarantee valid results. Respir Care 2010; 55: 689-94.

23. Derom E, Van Weel C, Liistro G, Buffels J, Schermer T, Lammers E, et al. Primary care spirometry. Eur Respir J 2008; 31: 197-203.

24. Steenbruggen I, Mitchell S, Severin T, Palange P, Cooper BG; on behalf of the Spirometry HERMES Task Force.

Harmonising spirometry education with HERMES: training a new generation of qualified spirometry practitioners across Europe. Eur Respir J 2011; 37: 479-81.

25. Pellegrino R, Viegi G, Brusasco V, Crapo RO, Burgos F, Casaburi R, et al. Interpretative strategies for lung function tests. Eur Respir J 2005; 26: 948-68.

26. Pérez-Padilla R, Vázquez-García JC, Márquez MN, Menezes AM; PLATINO Group. Spirometry quality-control strategies in a multinational study of the prevalence of chronic obstructive pulmonary disease. Respir Care 2008; 53: $1019-26$. 